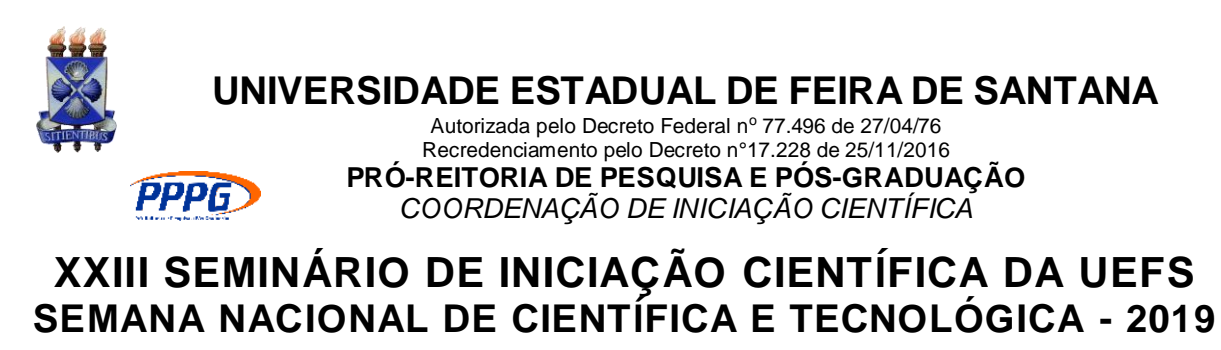

\title{
Acesso aos serviços de saúde por pacientes com diagnóstico de câncer de pulmão em Feira de Santana - BA
}

\author{
Gracas de Maria Dias Reis ${ }^{1}$; Juliana Alves Leite Leal ${ }^{2}$ \\ 1. Bolsista PIBIC/CNPq, Graduando em Medicina, Universidade Estadual de Feira de Santana, e-mail: \\ gmdiasreis@gmail.com \\ 2. Orientador, Departamento de Saúde, Universidade Estadual de Feira de Santana, e-mail: julianaleal@uefs.br
}

PALAVRAS-CHAVE: Acesso aos serviços de saúde; Direito à saúde; Sistema Único de Saúde.

\section{INTRODUÇÃO}

A saúde já foi conceituada de diversas formas no decorrer da história, de acordo com as características sociais, políticas, culturais e econômicas da sociedade em questão. Em 1948, a Organização das Nações Unidas, ao definir a saúde como "o estado do mais completo bemestar físico, mental e social e não apenas a ausência de enfermidade", ampliou o entendimento da saúde para além da presença de patologias. Em 1978, a Declaração de Alma-Ata criou as bases para que a Atenção Básica tivesse papel fundamental nos sistemas de saúde, que deveriam ser universais, inspirando diversos países a declararem o acesso à saúde como um direito universal (ANDRADE; ANDRADE, 2010).

No Brasil, até a promulgação da Constituição de 1988, o Estado Brasileiro, através do Ministério da Saúde, se responsabilizava pela prevenção agravos, através de programas de vacinação, e pelo funcionamento de alguns centros especializados, como ações para pessoas com tuberculose e acometimentos psiquiátricos. (ANDRADE; ANDRADE, 2010). Em 1986, a realização da $8^{a}$ Conferência Nacional de Saúde contou com a participação da sociedade civil e abordou a saúde como um direito, propondo reformulações para as formas de acesso aos serviços e lançando as bases que fundamentaram as resoluções da seção "Da Saúde" da Constituição de 1988. A Constituição Cidadã, através do Artigo 196, oficializou o princípio da "saúde como direito de todos e dever do Estado" (BRASIL, 2007).

O câncer de pulmão é o tipo de neoplasia maligna com maior incidência ao redor do mundo. Dados do Globocan, obtidos através da Agência Internacional de Pesquisa em câncer, um órgão da Organização Mundial da Saúde (OMS), indicam que, em 2018, foram registrados 2.093.876 novos casos de câncer de pulmão, correspondendo a 11,6\% do total de neoplasias. O câncer de pulmão é a neoplasia que mais mata ao redor do mundo, e, em 2018, foi responsável por 1761007 óbitos. (<http://gco.iarc.fr/today/data/factsheets/cancers/15Lung-fact-sheet.pdf $>$ ).

No Brasil, as neoplasias pulmonares também apresentam elevada incidência. Boing e Rossi (2007), ao estudarem a mortalidade e a distribuição regional de câncer traqueia, 
brônquios e pulmão país, no período de 1979 a 2004, encontraram 287484 óbitos (BOING; ROSSI, 2007).

Considerando o acesso aos serviços de saúde como direito universal e tendo em vista a alta prevalência do câncer de pulmão no Brasil, o presente estudo tem como objetivo geral discutir como tem se dado o acesso aos serviços de saúde por usuários/pacientes com diagnóstico de câncer de pulmão no município de Feira de Santana - BA, e como objetivos específicos descrever as estratégias utilizadas pelos usuários para obter acesso aos serviços de saúde para diagnóstico e tratamento do câncer de pulmão no município de Feira de Santana BA e identificar facilidades e dificuldades enfrentadas pelos usuários para obtenção de serviços/medicamentos/insumos de saúde para o tratamento do câncer de pulmão na rede de atendimento do Sistema Único de Saúde (SUS) em Feira de Santana - BA.

\section{METODOLOGIA}

O estudo teve caráter qualitativo e exploratório, sendo direcionado aos aspectos da realidade que não podem ser quantificados. O campo de estudo foi o município de Feira de Santana - BA, por ser a segunda cidade mais populosa do estado e primeira cidade do interior nordestino em população. O estudo foi realizado na Unidade de Alta Complexidade em Oncologia (UNACON). Os participantes foram divididos em dois grupos: I) Usuários/pacientes dos serviços de saúde com diagnóstico de Câncer de Pulmão, em tratamento, maiores de 18 anos e residentes de Feira de Santana; II) Trabalhadores dos serviços de saúde da UNACON há mais de 6 meses, como Médicos, Enfermeiros, Psicólogos, Assistentes Sociais, Nutricionistas, que atendem diretamente usuários/pacientes com Câncer de Pulmão. Os instrumentos utilizados para coletar os dados consistiram em dois roteiros de entrevista semi-estruturada, um para cada grupo. As entrevistas foram programadas de acordo com a disponibilidade dos participantes e foram realizadas no próprio serviço de saúde, em local reservado. Foram entrevistados quatro trabalhadores e seis usuários. Os participantes tiveram suas identidades protegidas e o anonimato garantido pelos pesquisadores durante todo o processo de coleta, análise e divulgação dos resultados do estudo. A análise de dados foi feita através da aplicação da Análise de Conteúdo, dividida em três etapas básicas: a pré-análise, a análise do material e o tratamento dos resultados (SANTANA; NASCIMENTO, 2010). Quanto às questões éticas, o presente estudo foi submetido ao Comitê de Ética e Pesquisa (CEP) da Universidade Estadual de Feira de Santana (UEFS) e respeitou os preceitos estabelecidos pela Resolução do Conselho Nacional de Saúde (CNS) no 466/12, pela Resolução do CNS no 580/18 e pela Resolução do CNS n ${ }^{\circ}$ 510 de 2016 (2016). Dessa forma, a coleta de dados aconteceu após autorização do CEP da UEFS, CAAE: 12304919.9.0000.0053, assim como posteriormente à concordância dos participantes, mediante a assinatura do Termo de Consentimento Livre e Esclarecido.

\section{RESULTADOS E/OU DISCUSSÃO}

O acesso aos serviços de saúde por pacientes com câncer de pulmão se dá em vários níveis de complexidade. Para acessar os serviços de diagnóstico e o tratamento, os usuários utilizaram diversas estratégias. Entre elas, podemos citar o uso de serviços privados, o acompanhamento por estudantes de Medicina da UEFS, na disciplina Práticas de Integração 
Ensino-Serviço-Comunidade (PIESC), a busca por favores de políticos e a influência de contatos na Secretaria de Saúde.

As principais dificuldades se relacionaram à necessidade de exames de alta complexidade para confirmação do diagnóstico. Tal desafio se manifestou tanto no que se refere ao processo de regulação na rede SUS, quanto à indisponibilidade de tais serviços na Unacon, o que retarda o início do tratamento.

"Porque a gente é unidade de alta complexidade e só aceita o paciente com diagnóstico fechado." (T1)

Houve convergência quanto a principal facilidade encontrada por esses usuários: o atendimento na Unacon. A presença do serviço de alta complexidade no interior, disponibilizando quimioterapia, radioterapia e braquiterapia, associada à infraestrutura do Hospital Dom Pedro de Alcântara e ao atendimento por uma equipe multiprofissional, é embasada pela Política Nacional de Atenção Oncológica, que preconiza que as Unidades de Alta Complexidade em Oncologia possam tratar os tipos mais prevalentes de neoplasias (BRASIL, 2005).

A discussão sobre acesso analisou cinco dimensões: disponibilidade, acessibilidade, acomodação funcional, capacidade financeira e aceitabilidade. A disponibilidade é compreendida como uma relação do volume de atendimento aos usuários e o tipo de necessidades; a acessibilidade é entendida como a relação entre a localização da oferta e dos usuários em seus territórios sociais; a acomodação funcional, percebida como a relação entre o modo como a oferta está organizada para aceitar os usuários e a capacidade/ habilidade dos usuários para se acomodarem a esses aspectos e perceberem sua conveniência; a capacidade financeira é entendida como relação entre os custos dos serviços; e a aceitabilidade é compreendida como a relação entre as atitudes dos usuários sobre os trabalhadores de saúde e as práticas dos serviços, como também a aceitação dos trabalhadores e dos serviços em prestar assistência a esses usuários (THEIDE; AKEWENGO; MCINTYRE, 2014).

No que se refere à disponibilidade, o atendimento no Hospital Dom Pedro de Alcântara oferta exames laboratoriais e de imagem gratuitos para os usuários com câncer de pulmão, além do serviço de internamento. O principal desafio está relacionado à falta de equipe de cirurgia torácica na Unacon, o que impede a realização de procedimentos de estadiamento e terapêuticos, além de gerar a necessidade de deslocamento para Salvador.

"Hoje, como não tem a equipe ainda, se a recepção percebe que é uma demanda pra cirurgia, ela nem atende, nem atende esse paciente. Ela já orienta que vá procurar o Aristides." (T1)

Em relação à acessibilidade, houve convergência quanto à adequação da localização, disponibilidade de cadeiras de rodas, ausência de escadas e possibilidade de receber quimioterapia em leitos. No quesito acomodação funcional, a grande quantidade de usuários que recebe assistência na Unacon, que recebe pacientes de mais de 70 municípios, gera superlotação da recepção, sendo sugerida, tanto por trabalhadores quanto por usuários, a ampliação do espaço. A capacidade financeira tem grande impacto nos processos de diagnóstico e estadiamento, quando há maior necessidade de exames. Nesse período, os usuários recorrem à venda de bens, a empréstimos e à ajuda financeira de familiares para custear exames. 
"Conseguir fazer os exames demorou um pouco porque eu não tive o dinheiro, minha esperança foi meu marido. Ele pegou do dinheiro dele, me ajudou bastante, foi que eu fiz os exames." (P2)

Apesar de o tratamento ser gratuito, os gastos com medicamentos, principalmente para manejo da dor, se tornam expressivos. Quanto à aceitabilidade, todos entrevistados citaram o relacionamento entre trabalhadores e usuários como principal ponto positivo do processo terapêutico. A atitude dos profissionais foi caracterizada como sensível e proativa. $\mathrm{O}$ relacionamento entre os usuários foi citado enfatizando a paciência com os mais debilitados.

\section{CONSIDERAÇÕES FINAIS}

O estudo pôde concluir que o acesso aos serviços de saúde por pacientes com câncer de pulmão em Feira de Santana teve grandes avanços desde o surgimento da Política Nacional de Atenção Oncológica. Serviços diagnósticos e terapêuticos, equipe multiprofissional e atendimento hospitalar estão presentes na rede SUS. No entanto, há desafios a serem enfrentados para que o acesso possa ocorrer de forma efetiva para todos os usuários, garantindo o acesso ao diagnóstico precoce e gratuito e fornecendo condições para que o tratamento ocorra de forma facilitada.

\section{REFERENCIAS}

ANDRADE, E. N., ANDRADE, E. O. O SUS e o direito à saúde do brasileiro: leitura de seus princípios, com ênfase na universalidade da cobertura. Revista Bioética, v. 18, n. 1, p. 61-74, 2010.

BRASIL. Ministério da Saúde. Política Nacional de Atenção Oncológica. Brasília, DF, 2005 BRASIL. Ministério da Saúde. Secretaria de Gestão Estratégica e Participativa. Departamento de Apoio à Gestão Participativa. Caminhos do direito à saúde no Brasil. Brasília, DF, 2007. BOING, A. F., ROSSI, T. F., Tendência temporal e distribuição espacial da mortalidade por câncer de pulmão no Brasil entre 1979 e 2004: magnitude, padrões regionais e diferenças entre sexos. Jornal Brasileiro de Pneumologia, v. 33, n. 5, p. 544-551, 2007.

GLOBOCAN: The Global Cancer Observatory. Lyon, 2019. Disponível em: $<$ http://gco.iarc.fr/today/data/factsheets/cancers/15-Lung-fact-sheet.pdf $>$. Acesso em: 12 jul. 2019.

SANTANA, J. S. S., NASCIMENTO, M. A. A.; Pesquisa: métodos e técnicas de conhecimento da realidade social. Feira de Santana: Universidade Estadual de Feira de Santana, 2010.

THIEDE, M.; AKEWENGO, P.; MCINTYRE, D. Explorando as dimensões do acesso. In: MCINTYRE, D.; MOONEY, G. Aspectos econômicos da equidade em saúde. Rio de Janeiro: Editora Fiocruz, 2014. 